\title{
Next of kin's Reactions to Results of Functional Neurodiagnostics of Disorders of Consciousness: a Question of Information Delivery or of Differing Epistemic Beliefs?
}

\author{
Katja Kuehlmeyer (D) Andreas Bender • Ralf J. Jox • \\ Eric Racine • Maria Ruhfass • Leah Schembs \\ Received: 19 January 2021 / Accepted: 7 March 2021 / Published online: 24 March 2021 \\ (C) The Author(s) 2021
}

\begin{abstract}
Our recent publication in Neuroethics reconstructed the perspectives of family caregivers of patients with disorders of consciousness (DOC) on functional neurodiagnostics (Schembs et al., Neuroethics, 2020). Two papers criticized some of our
\end{abstract}

K. Kuehlmeyer $(\bowtie) \cdot$ M. Ruhfass $\cdot$ L. Schembs Institute of Ethics, History and Theory of Medicine, LMU Munich, Lessingstr. 2, 80336 Munich, Germany

e-mail: katja.kuehlmeyer@med.lmu.de

A. Bender

Department of Neurology, University Hospital, LMU Munich, Marchioninistr. 15, 81377 Munich, Germany

\author{
A. Bender \\ Therapiezentrum Burgau, Kapuzinerstraße 34, 89331 Burgau, \\ Germany
}

R. J. Jox

Clinical Ethics Unit and Institute of Humanities in Medicine, Lausanne University Hospital and University of Lausanne, Rue du Bugnon 21, CH-1011 Lausanne, Switzerland

\section{R. J. Jox}

Service of Palliative and Supportive Care, Department of Medicine, Lausanne University Hospital, Avenue Pierre-Decker 5, CH-1011 Lausanne, Switzerland

E. Racine

Pragmatic Health Ethics Research Unit, Institut de recherches cliniques de Montréal (IRCM), 110 Pine Ave W, Montreal, Québec H2W 1R7, Canada methodological decisions (Peterson, Neuroethics, 2020; Andersen et al., Neuroethics, 2020) and commented on some conclusions. In this commentary, we would like to further explain our methodological decisions. Despite the limitations of our findings, which we readily acknowledged, we continue to think they entail valid hypotheses that need further investigation. We conclude that some caregivers with high hopes for the recovery of their loved ones with DOC will most likely not consider results of functional neuroimaging as guiding information for treatment decisions, despite efforts taken to deliver information to them. Caregivers of that type might argue that such test-results are not a reliable source of information for the judgement of whether their loved one is likely going to recover or not (prognosis). We introduce the concept of epistemic beliefs to formulate this hypothesis and suggest that future qualitative studies in this area should be aware of such beliefs when investigating the effects of functional neurodiagnostics on knowledge communication and shared decision making for patients with DOC.

Keywords Unresponsive wakefulness syndrome (UWS) · Minimally conscious state (MCS) · Functional neuroimaging (fMRI) - Electroencephalography (EEG) . Surrogate decision making $\cdot$ Shared decision making

\section{Introduction}

In our recent publication in Neuroethics, we reported the results of a qualitative study with next of kin of adult 
patients with disorders of consciousness (DOC), including patients with unresponsive wakefulness syndrome (UWS) and patients in the minimally conscious state (MCS) [1]. We investigated whether next of kin would consider the results of functional neurodiagnostics in their reasoning about treatment decisions for patients with DOC by exploring their portrayal of neurodiagnostic measures at one rehabilitation center. Functional neurodiagnostics includes two different types of measures: imaging tests or scans (e.g. functional magnetic resonance imaging (fMRI)) and electrophysiological measurements (e.g. electroencephalography (EEG)). They allow researchers and clinicians to examine changes in brain activity at defined localizations and time points and are used to detect hidden signs of awareness [2]. The approach employed in the rehabilitation center in our study was a high definition (HD)-EEG paradigm which follows recent European guideline recommendations on the diagnosis of DOC [3].

The results of this HD-EEG examination was shown to be a topic of little importance to our participants. It was considered as just one of a variety of instruments for the professional evaluation of the patient's current state and as not more persuasive than other clinical examination. Regardless of the HD-EEG results, all participants in our study remained optimistic towards their loved ones meaningful recovery. We tried to explain this observation through a psychological information processing and coping model.

Two commentaries, while complementing our efforts, challenged our study report $[4,5]$. While most of these limitations were already acknowledged in our publication, the authors elaborated upon some issues and made suggestions for improvements for further studies. In addition, they questioned whether our study would have produced different findings if we had applied different methods. It is very probable that other studies will produce somewhat differing findings on that matter. Our study has been conducted in a specific cultural and organizational context and whether it can be generalizable to other caregivers in other contexts is yet to be seen. It was the first publication on the issue that we know of and it followed an exploratory approach. Its sample is small, yet acceptably large for a qualitative study and based on a preselection carried through a recruitment process using gatekeepers in a rehabilitation center.

The commentaries raise important questions: Can our results be disregarded because of their methodological limitations? Or is there a type of family caregiver that considers information about neurodiagnostics in ways that will not inform their attitudes towards treatment decisions for patients with DOC by functional neurodiagnostics? Should that change the ways experts in the field consider the effects of these measures on patients' families and surrogate decision makers [6]? Should we take different precautions during the process of their implementation in routine care?

First, we will briefly reiterate our claims and reply to the key points, thereby further explaining the reasoning behind our choices. Second, we will suggest a concept that future studies should pay attention to when investigating the reactions of patients with DOC's next of kin to functional neurodiagnostics measures: the concept of epistemic beliefs [7]. More research - empirical as well as conceptual - should shed light on the underlying questions at the core of this discussion.

\section{Reply to Criticism Concerning the Research Methods of our Study}

The commentaries underscore both methodological considerations about data collection (i.e. selection of participants, timing of the data collection (recall bias), method of data collection, desire for different/additional data) and the transferability of this study to other studies on the effects of functional neurodiagnostics and knowledge communication $[4,5]$.

Methodological Considerations of Data Collection

\section{Selection of Participants}

Andersen et al. criticized the sample selection of our study, especially with regard to the exclusion of next of kin that considered terminating life-sustaining treatment in the aftermath of the disclosure of the results of the neurodiagnostics [4].

For this study, we were looking for participants that had experience with functional neurodiagnostics, which is rarely accessible in rehabilitation centers in Germany. They have not yet been integrated in standard care. The European guideline recommendations on the diagnosis of DOC have not yet been transferred to German guidelines [3]. A German guideline for the neurorehabilitation of adult patients with DOC commenced in 2019 with an expected completion date of 2021. It will however focus 
on therapy but not diagnostics [8]. If a rehabilitation center offers functional neurodiagnostics, it seldom has access to expensive technologies like MRI or positron emission tomography (PET) and it is rarely conducting its own research similar to university hospitals. The possibility of a collaboration with a center that conducts cuttingedge studies in this field was only possible due to the good working relationships of two of our co-authors.

Within the center, we relied on study gatekeepers and clinical gatekeepers to inform potential participants about the interview study, which has led to a double pre-selection: 1) only the next of kin of participants who were eligible for their neuroscientific study (for example, excluding patients who were sedated, colonized by multiresistant bacteria, considered vegetatively instable; patients in the process of being discharged soon or cases where patients and caregivers were not able to speak proficient German) and 2) only the next of kin they could approach and that they allowed us to meet were presented to us (gatekeeper effect).

The selection of patients who may benefit the most from the results of functional neurodiagnostics are those who are likely to show signs of hidden awareness that were not detected by behavioral observation. Andersen mentioned that the "clinical rationale for the EEG assessment is the potential to uncover covert awareness, and thereby change a diagnosis from the detrimental diagnosis of Unresponsive Wakefulness State (UWS) to the more promising diagnosis of Minimally Conscious State." The neurodiagnostic examination was not limited to patients that had the potential for discovering hidden awareness (prior diagnosis of UWS), but also included patients with behavioral signs of awareness (prior diagnosis of MCS). Since functional neurodiagnostic methods are still in the process of being tested, refined and validated, it was important for the neuroscientific research team to also examine patients who showed signs of consciousness already on a clinical level (MCS). In order to confirm that by use of the HDEEG researchers and clinicians can actually distinguish MCS from UWS. One further aim of the neuroscientific study was to test the reliability of functional neurodiagnostics as a predictor of further improvements compared to other approaches of diagnostics (e.g. behavioral assessment), i.e. is the HD-EEG informative about future improvement from UWS to MCS or from MCS to emergence from MCS (eMCS). This aim most likely applies to its usage in ongoing research. In future clinical care resources might be limited to unclear cases.
If we had limited our participants of the interview study to those cases with a prior diagnosis of UWS, we would only have included five of the seven cases. In only one case, the family caregiver reported a shift of the diagnostic category after the examination (from UWS to MCS). In that case, the subjective impression of the family caregiver that her husband is aware and that he will be able to benefit from further rehabilitative attempts has been confirmed. Her attitude towards the rehabilitation potential of the patient was continuously optimistic. However, the participant described a benefit: The rehabilitation efforts of the medical staff had been intensified by the chance of further recovery associated to the new category (MCS). Another group of caregivers that could "benefit" considerably from functional neurodiagnostics could be those where the test results confirm the diagnosis of UWS. This happened to at least one participant in our study, but instead of changing the perspective of the next of kin (that her daughter was not aware as she assumed), she questioned the validity of the examination and argued for a second test to be taken.

What Andersen et al. mostly refer to is the decision not to follow-up on family caregivers who, in light of the recent diagnosis/prognosis, decided for the option of pursuing palliative care. Our clinical gatekeeper decided to exclude these families for research ethical concerns. First, they were worried about a potential harm of the study as they did not want to overburden the next of kin with an interview in cases where a redirection of the goal of care was discussed. Caregivers furthermore might have felt the need to justify their decisions to us which could potentially lead to feelings of guilt of uncertain duration. Second, the clinical gatekeeper wanted to prevent a mistaken impression that the functional HD-EEG examination as a method of still unknown reliability was decisive about life or death. Furthermore, the gatekeeper worried that the rehabilitation center might have been seen in a bad light, in the event that we misrepresented the case in our study or if the media misrepresented our study publicly, e.g. as a measure to detect "a life worth living" and relate it to the Nazi crimes during the 1930s and 40s. With relation to that potential for misunderstanding, it is important to note that in clinical practice decisions to redirect the treatment strategy towards palliative care are complex judgements that cannot be based on one diagnostic test result alone. The test result could be used to inform diagnosis and prognosis, but still a clinical assessment would predominantly be based on the observed clinical course of the patient. 
At some point it will be crucial to include those cases in studies like ours to get insight in the full spectrum of possibilities of effects of functional neurodiagnostics but at such an exploratory stage, we considered it acceptable to not cover the full spectrum of possible cases and be transparent about the preselection of the cases. It is likely that other researchers in clinical environments, in other contexts, would need to also strike such kinds of compromises. This is both a strength and a limitation of non-experimental research about the actual uses of technology.

Hypothetically speaking, if we had one participant who based their attitude towards the appropriate treatment strategy on functional neurodiagnostics, what difference would that have made for the reporting of our study results? At that point we would like to remind readers that with qualitative research we do not attempt to gather representative findings about a distribution of attributes within a certain population. Our aim was to describe cases that we selected systematically in a certain time frame and the findings suggest that there could be a type of next of kin who ignores neurodiagnostic findings. Family members of that type seem not to question their own beliefs about the patient's condition or rehabilitation potential based on diagnostic results. This does not exclude that there may be other types of next of kin that react to functional neurodiagnostics differently.

As a last remark, patients at a rehabilitation center are already a pre-selected sample as the patient's admission is based on the presumption that further improvement is possible and a caregiver's hope might play a crucial role in decisions to keep a patient in or re-admit a patient to such a health care facility. Recruiting through a rehabilitation facility means excluding patients whose health insurances do not pay for specialized rehabilitation, patients in regions where these facilities are not available, patients whose family caregivers, do not overcome the challenges of applying for rehabilitation for their loved one and family caregivers who care for the patients themselves at home because of losing trust in the health care system altogether.

\section{Timing of the Data Collection: Recall Bias}

Andersen et al. furthermore address the large time gaps between the EEG examinations and the interviews (between 1 and 9 months) which might have led to recall bias [4]. At the time of the interview, being under the impression of the current situation might have made it impossible for the participants to go back in time and recall how they had reacted to the test results. This criticism holds merit, and we regret that we could not have been speaking to the next of kin earlier. Our qualitative study was not fully implemented in the routines of the rehabilitation center and the recruitment of potential participants proceeded slowly. We may have missed out short-term-effects of disclosure of medical information that were forgotten in the meantime. In a current qualitative study within the PerBrain-Project (German BMBF-Funding Number: 01KU2003), we will implement a different integration strategy and plan to conduct interviews within 1 to 2 weeks after the results of the functional neurodiagnostics examination have been disclosed [9].

\section{Method of Data Collection}

After addressing the exclusion of cases where the goal of care was shifted to comfort care only, Andersen et al. suggest a different basis for data collection than interviews, namely what they referred to as "co-presence", a form of participant observation (or observatory participation) as part of an ethnographic research approach [4]. They adopted this method in an ongoing anthropological study that aims at investigating "knowledge-making among clinicians and researchers" in light of the development of new neurodiagnostics tools for patients with DOC [4]. It seems, in the study that Andersen et al. are conducting, trust is built and researchers are granted access to all areas of care.

Contrary to Andersen et al., we do not think that datagathering through observation and co-presence is necessarily the best or the only way to deal with the addressed problem. Familiarization with potential participants without directed observations and note taking, like being present and being introduced during clinical routines based on a closer working relationship between the interviewers and the clinical team could also result in meaningful opportunities to build relationships with family caregivers early on. It could also be an opportunity to conduct multiple interviews throughout the course of the stay. There are also ethical concerns with ethnographic research. One example is the concern that participants may not fully capture what consenting to observation means. While having casual conversations with researchers participants may forget that they are giving them private information for a qualitative study. 
This could result in a feeling of deception. To prevent this, the method and the gathered data should be carefully explained to participants (e.g. in the form of giving participants the opportunity to repeatedly review the gathered data).

Proposal of an experimental approach for data collection Peterson criticizes that we did not examine the attitudes of family caregivers towards EEG assessment before seeking-out the participation in the neurodiagnostic study [5]. He expects them to fall into two groups, 1) those patients for whom next of kin have chosen the rehabilitation center with the intent to get neurodiagnostic tests done or 2) those patients who were passively enrolled in the studies where the test was performed without further knowledge of the meaning of functional neurodiagnostics. Peterson assumes that such attitudes might have an effect on how the EEG results are evaluated and processed by the participants. The underlying hypothesis could be: Those who seek out the test are more likely to attribute greater meaning to the findings. Furthermore, both point to the issue of how the EEG results were communicated to the participants based on a standardization in the delivery of the EEG results $[4,5]$. They argue that the way this information is delivered might have an impact on how it is perceived by the next of kin. We interpret these suggestions as recommendations within a quasi-experimental research approach.

The clinical neurodiagnostics study that was performed at the neurorehabilitation center did not include an examination of its effects on the caregivers. Since our goal was to study existing practices it would have been counterproductive if we had asked for a standardization of the communication of the test results. A qualitative interview study does not have to follow a controlled experimental design with the purpose of testing the effect of an intervention (functional neurodiagnostics/ disclosure of their results). Other than a quantitative/ nomothetic design, a qualitative research design gives researchers the opportunity to explore the every-dayexperiences of family caregivers from their perspectives. A controlled experimental study with the aim of measuring the effects of the intervention (disclosure of results of neurodiagnostics) would for example investigate the attitude prior to the test, follow a standardized protocol with a standardized delivery of information, compare it with the attitude after the test and report the amount of attitude alteration.
We did not want to influence or manipulate the setting but get an insight into the natural considerations of participants in light of their experiences with patient care and these specific medical evaluations during their stay at the rehabilitation center. Our goal was to understand the complexity of the situation rather than to explain a certain aspect of it. We aimed at reconstructing the subjective perspectives of family caregivers at a certain point in time. The context of the case can be used to explain described phenomena, but we did not try to standardize the research, based on prior hypotheses, with the aim to make generalizable statements.

What is meant by using the context of the case to explain its results can be shown in the following example: one of our participants (participant 1) mentioned that she had chosen this rehabilitation center so that functional neurodiagnostics could be conducted to investigate whether her daughter (diagnosed with UWS) would show signs of hidden awareness. She was well informed about the HD-EEG approach in the center and its role in the diagnostis of DOC. This participant was sure her daughter was aware without being able to show it to others. She wanted to use the findings for the purpose of obtaining more appropriate care for her daughter. The results of the tests showed no signs of hidden awareness, which the participant however did not accept as a conclusive result. She assumed that her daughter's tiredness at the time of the test was responsible for the allegedly false test results. She insisted that the test be repeated. In that case, although the participant had chosen the rehabilitation center because of the test (high expectation), the test result did not change her belief in that her daughter was conscious and would recover (no change of attitude), but based on her attitude she invested more effort to change the test result (more engagement in care). We tried to explain that by our information processing and coping model. The result that is derived from understanding that case in that way is a first hypothesis that could be tested in further studies, not already the attempt to prove that this often is the case.

Transferability of this Study to Other Studies on the Effects of Functional Neurodiagnostics and Knowledge Communication

This leads us to the question of whether we can transfer the results of this study to other contexts. Peterson mentions that functional neurodiagnostics is an 
umbrella term, representing a spectrum of possible measures, where the HD-EEG represents only one example of the available investigational techniques used to assess brain-injured patients. Hence, our findings cannot speak to the effect of other imaging modalities as they have different purposes, a different base of evidence and represent consciousness differently.

We agree that methods of functional neuroimaging like fMRI and certain forms of PET lend themselves to more visual rendition of results even though both are heavily based on reconstruction of data into an image. These could have been experienced and remembered differently by family caregivers. Yet, in the study in the rehabilitation centre, HD-EEG results were also presented graphically in the reports by means of graph theory in order to visualize brain connectivity, based on Chennu et al. [10].

It might be derived from some of the above mentioned criticism that there should be more effort to make family caregivers understand the validity of such objective findings with more significant results and better medical education. This leads us to the central question of our commentary whether other studies may come to differing results. If they report a high hope for the recovery of a patient with DOC, what effect will the results of functional neurodiagnostics have on the attitudes of next of kin towards the patient's current state (diagnosis) and possible future (prognosis)? Before we address this question more directly, we would like to suggest a theoretical concept that could be useful in studies like ours: the concept of epistemic belief.

\section{Epistemic Beliefs and Informational Needs of Next of Kin of Patients with DOC}

Epistemic beliefs have become a target of increased research interest in educational psychology, but so far we have not identified its usage in the neuroethics of DOC, although there has been an ongoing discussion about lay understandings of neuroscience and related methodologies, including in the condition of DOC $[7$, 11-15]. Epistemic beliefs are defined as "a person's beliefs about the nature of human knowledge, like its certainty and how it is conceptualized, and a person's beliefs about the criteria for and the process of knowing" (online resource without pages) [7]. It describes the personal assumptions and own pre-structuring of how one encounters the cognizable world. The concept has been used in quantitative studies to examine lay-people's epistemic beliefs about medicine as a prerequisite for shared-decision-making [16].

In our research area, epistemic beliefs could be investigated with respect to how family caregivers perceive functional neurodiagnostics as a reliable source of knowledge about whether the patient is aware of their surroundings (diagnosis) and whether they have potential for further recovery (prognosis). Based on our research and clinical experience with caregivers of patients with DOC, we hypothesize that while a majority of medical professionals (especially doctors, neuropsychologists, etc.) rely on the measurability of signs of awareness, there are next of kin who rather rely on the perceived (ongoing) relationships with the patient when making their judgment about the current state and possible future of the patient. In our current qualitative study within the PerBrain project, we currently explore how the informational needs and dealing with information of family caregivers of patients who undergo functional neurodiagnostics are influenced by their epistemic beliefs. We believe other studies in this area should also pay attention to this concept. We suspect that it will be crucial in differentiating family caregivers who will be willing to change their attitudes towards the evaluation of the patient's current state and potential for improvement in the future based on functional neurodiagnostics from those who will not be willing to do that, despite refined technologies and improved information communication through brochures, decision-aids or other informational interventions.

\section{Conclusion}

Qualitative research can shed light on the meaning of functional neurodiagnostics from the perspective of family caregivers, but the research in this area is just starting and is far from being conclusive. Future studies should examine epistemic beliefs to explore whether it can explain cases were neurodiagnostics results are not assigned meaning by family caregivers. If epistemic beliefs play a role in the communication with family caregivers of next of kin, an approach of multidirectional knowledge discussion, instead of unilateral knowledge delivery, should be considered. 
Funding Open Access funding enabled and organized by Projekt DEAL.

\section{Declaration}

Conflict of Interest The authors declare no conflicts of interest related to this publication. The publication that it refers to presented research that was funded by the Friedrich-Baur-Stiftung (Grant Number: $12 / 14$, funding period $8 / 2014$ to $07 / 2016$ ). This article is part of LS's cumulative dissertation (Dr. med.) at the Medical Faculty of LMU Munich. LS received funding by the MaxWeber-Program during her work at this study.

Open Access This article is licensed under a Creative Commons Attribution 4.0 International License, which permits use, sharing, adaptation, distribution and reproduction in any medium or format, as long as you give appropriate credit to the original author(s) and the source, provide a link to the Creative Commons licence, and indicate if changes were made. The images or other third party material in this article are included in the article's Creative Commons licence, unless indicated otherwise in a credit line to the material. If material is not included in the article's Creative Commons licence and your intended use is not permitted by statutory regulation or exceeds the permitted use, you will need to obtain permission directly from the copyright holder. To view a copy of this licence, visit http://creativecommons.org/licenses/by/4.0/.

\section{References}

1. Schembs, L., M. Ruhfass, E. Racine, R.J. Jox, A. Bender, M. Rosenfelder, and K. Kuehlmeyer. 2020. How does functional Neurodiagnostics inform surrogate decision-making for patients with disorders of consciousness? A Qualitative Interview Study with Patients' Next of Kin. Neuroethics. https://doi.org/10.1007/s12152-019-09425-4.

2. Aguirre, G.K. 2014. Functional neuroimaging: Technical, logical, and social perspectives. Hastings Cent Rep Spec no: S8-18. https://doi.org/10.1002/hast.294.

3. Kondziella, D., A. Bender, K. Diserens, W. van Erp, A. Estraneo, R. Formisano, S. Laureys, L. Naccache, S. Ozturk, B. Rohaut, J.D. Sitt, J. Stender, M. Tiainen, A.O. Rossetti, O. Gosseries, C. Chatelle, and the EAN Panel on Coma, Disorders of Consciousness. 2020. European academy of neurology guideline on the diagnosis of coma and other disorders of consciousness. European Journal of Neurology 27 (5): 741-756. https://doi.org/10.1111 /ene.14151.

4. Andersen, L.M., H.B. Boelsbjerg, and M.T. Høybye. 2020. Disorders of Consciousness: An Embedded Ethnographic Approach to Uncovering the Specific Influence of Functional Neurodiagnostics of Consciousness in Surrogate Decision Making. Neuroethics. https://doi. org/10.1007/s12152-020-09448-2.
5. Peterson, A. 2020. How will families react to evidence of covert consciousness in brain-injured patients? Neuroethics. https://doi.org/10.1007/s12152-019-09428-1.

6. Jox, R.J., J.L. Bernat, S. Laureys, and E. Racine. 2012. Disorders of consciousness: Responding to requests for novel diagnostic and therapeutic interventions. Lancet Neurology 11 (8): 732-738. https://doi.org/10.1016/S14744422(12)70154-0.

7. Kienhues, D. 2013. Epistemic Beliefs. In Oxford Bibliographies (Education section), ed, ed. L.H. Meyer. New York: Oxford University Press.

8. AWMF. 2019. Neurologische Rehabilitation bei Koma und schwerer Bewusstseinsstörung im Erwachsenenalter. S3 guidline, registration number 080-006.

9. Bender, A. 2020. PerBrain - Multimodale Untersuchungen für die personalisierte Diagnose und Prognose von Patienten mit schweren Bewusstseinsstörungen nach akuter erworbener Hirnschädigung. https://www.gesundheitsforschung-bmbf. de/de/perbrain-multimodale-untersuchungen-fur-diepersonalisierte-diagnose-und-prognose-11840.php. Accessed 18 Jan 2021.

10. Chennu, S., J. Annen, S. Wannez, A. Thibaut, C. Chatelle, H. Cassol, G. Martens, C. Schnakers, O. Gosseries, D. Menon, and S. Laureys. 2017. Brain networks predict metabolism, diagnosis and prognosis at the bedside in disorders of consciousness. Brain 140 (8): 2120-2132. https://doi. org/10.1093/brain/awx163.

11. Boyd, E.A., B. Lo, L.R. Evans, G. Malvar, L. Apatira, J.M. Luce, and D.B. White. 2010. "It's not just what the doctor tells me:" factors that influence surrogate decision-makers' perceptions of prognosis. Critical Care Medicine 38 (5): 1270-1275. https://doi.org/10.1097/CCM.0b013e3181d8 a217.

12. Racine, E., O. Bar-Ilan, and J. Illes. 2005. fMRI in the public eye. Nature Reviews. Neuroscience 6 (2): 159-164. https://doi.org/10.1038/nrn1609.

13. Racine, E., S. Waldman, J. Rosenberg, and J. Illes. 2010. Contemporary neuroscience in the media. Social Science \& Medicine 71 (4): 725-733. https://doi.org/10.1016/j. socscimed.2010.05.017.

14. Phipps, E.J., M. Di Pasquale, C.L. Blitz, and J. Whyte. 1997. Interpreting responsiveness in persons with severe traumatic brain injury: Beliefs in families and quantitative evaluations. The Journal of Head Trauma Rehabilitation 12 (4): 52-69.

15. Gray, K., T.A. Knickman, and D.M. Wegner. 2011. More dead than dead: Perceptions of persons in the persistent vegetative state. Cognition 121 (2): 275-280. https://doi. org/10.1016/j.cognition.2011.06.014.

16. Kienhues, D., and R. Bromme. 2012. Exploring laypeople's epistemic beliefs about medicine - A factor-analytic survey study. BMC Public Health 12 (1): 759. https://doi. org/10.1186/1471-2458-12-759.

Publisher's Note Springer Nature remains neutral with regard to jurisdictional claims in published maps and institutional affiliations. 